\title{
Effects of the German 1993 health reform law upon primary care practitioners' individual performance: results from an empirical study in sentinel practices
}

\author{
M Hoopmann, F W Schwartz, J Weber
}

\begin{abstract}
An empirical study in Lower Saxony aimed to investigate any changes in primary care physicians' diagnostic and therapeutic strategies as a result of Germany's 1993 health reform act (known as the Gesundheitsstruktur Gesetz or GSG), which included the countrywide implementation of a strict drug budget. A sentinel network consisting of a $37 \%$ sample of 350 randomly selected doctors $(n=130$, GPs, general internists) was established in Lower Saxony. Four cross sectional surveys, each focussing on one group of health problems, were carried out during 1993 . These aimed to show whether sentinel practice networks are suitable for reporting physicians' attitudes towards health care cost containment policies and, secondly, changes in physicians' quantitative and qualitative assessments of the 1993 reform act during its first year of implementation. Participating physicians reported patient consultations $(n=3728)$. Standardised questionnaires ascertained sociodemographic variables and major reasons for the patients' visit. Data on the diagnoses associated with the patient's main reason for the consultation, the doctor's assessment of the severity of the problem, and diagnostic and treatment strategies were also recorded. The questionnaire focussed on changes in therapy made by the physician together with the reasons for these changes. A number of treatment changes made with regard to cost containment were recorded. During the course of 1993 a decrease in reported changes in treatment was noticed. As expected, some doctors recorded a reduction in successful outcomes of treatment and ascribed this to the reform act. Differences between the four surveys with regard to the influence of the health reform act on the frequency of changes in treatment and the physicians' expectations cannot be explained sufficiently by the physicians' adaptation to the cost containment policies within the year.
\end{abstract}

(FEpidemiol Community Health 1995;49(Suppl 1):33-36)

The paramount objective of the practice of medicine is to provide quality care. ${ }^{1}$ The costs of assuring quality within ambulatory medical care is of major current concern. In the past decade numerous efforts have been made to control expenditure in the German health care system. The 1989 reform act (the Gesundheitsreform Gesetz or GRG) laid a successful foundation for a structural overhaul of Germany's health system, which culminated in the 1993 reform act (Gesundheitsstruktur Gesetz or GSG). There has been much debate over the impact (positive or negative) of implemented reforms, such as the strict drug budget, on the performance of ambulatory care. ${ }^{23}$ Doctors have found their decisions challenged by administrators. If physicians' national prescribing of drugs exceeds DM $24 \cdot 1$ billion, they are held collectively liable for the first DM 280 million ( $\$ 134$ million through the sickness fund physician associations, with the remainder taken out of future fee reimbursements). ${ }^{4}$ At the beginning of 1993 both physicians and patients within the primary care sector felt equally insecure.

Despite an extensive primary care system, there are no data from empirical scientific investigations on the effects that health reform laws have upon individual medical practice. There is also a lack of information on physicians' attitudes towards cost containment policies in Germany. In addition, although sentinel networks (usually working on epidemiological morbidity data) have been successfully developed in several European countries, ${ }^{56}$ no sentinel research surveys dealing with health policies have been published.

A sentinel project was initiated in January, 1993 in north western Lower Saxony. It aimed to provide data on the patient management of practice based primary care physicians with regard to the recent cost containment policies. The goals were, firstly, to determine whether sentinel practice networks are a suitable mechanism for reporting physicians' attitudes towards health care cost containment policies and, secondly, to illustrate any change in the doctors' quantitative and qualitative assessments of the 1993 reform act during the course of the first year of implementation.

\section{Methods}

The sentinel network consists of a $37 \%$ sample of 350 randomly selected primary care physicians $(n=130)$. The sampling frame was derived from the lists of the regional associations of sickness fund physicians. The study was 
carried out in the region of north western Lower Saxony. Here, most of the important sociodemographic features and the structure of ambulatory medical care are similar to those elsewhere in Lower Saxony. To achieve the final sample, 350 doctors (GPs, general internists) were selected randomly. The sample was stratified in relation to region and speciality, and the sampling procedure produced a representative sample of practices in the study region.

Data derived from Lower Saxony's Association of Sickness Fund Physicians were considered as an instrument of validation. The distributions of age, sex, practice speciality, and the location of pratices (urban or rural) showed that there was no systematic bias in the sampled doctors compared with the total number of primary care practitioners in Lower Saxony.

Efforts were made to reduce non-response, including a token payment in compensation for the time taken.

The sentinel practitioners participated in at least one of the four different cross sectional surveys conducted in May, June, October, and November 1993. In each of the two week surveys the participants were asked to report patient consultations in which one of the following common symptom complexes was given as a major reason for visit:

- Group 1: vertigo, low blood pressure, fatigue, insomnia, disorders of coordination, restlessness, anxiety, and loss of memory in patients between 50 and 79 years of age. This survey was carried out in May, 1993.

- Group 2: leg problems - paresthesias, "restless legs", oedema, venous thrombosis, and eczema in patients over 20 years of age. This survey was carried out in June, 1993.

- Group 3: chronic joint pains due to rheumatic conditions in patients aged 50 to 79 years. This survey was carried out in September/October, 1993.

Table 1 Data collection in the study

\begin{tabular}{lcc}
\hline Survey complex & $\begin{array}{l}\text { No of contact report } \\
\text { forms completed }\end{array}$ & $\begin{array}{l}\text { No of reporting } \\
\text { physicians }\end{array}$ \\
\hline Group 1 - vertigo etc. & 688 & 58 \\
Group 2 - leg problems & 735 & 68 \\
Group 3 - joint pains & 1026 & 89 \\
Group 4 - gastrointestinal disturbances & 1279 & 86 \\
Total & 3728 & 130 \\
\hline
\end{tabular}

Table 2 Sex distribution of the patients included in the study

\begin{tabular}{lll}
\hline Survey complex & Men (\%) & Women (\%) \\
\hline Group 1 - vertigo etc. & 34.5 & 65.5 \\
Group 2 - leg problems & $28 \cdot 3$ & 71.7 \\
Group 3 - joint pains & 44.5 & 55.5 \\
Group 4 - gastrointestinal disturbances & 47.7 & 52.3 \\
Total & 40.5 & $59 \cdot 5$ \\
\hline
\end{tabular}

Table 3 Number of changes in treatment and proportion of changes in treatment explained by the health reform act

\begin{tabular}{lll}
\hline Survey complex & $\begin{array}{l}\text { Total no of changes } \\
\text { in treatment }\end{array}$ & $\begin{array}{l}\text { Proportion of treatment changes } \\
\text { explained by health reform (\%) }\end{array}$ \\
\hline Group 1 - vertigo etc. & 185 & 41 \\
Group 2 - leg problems & 165 & 21 \\
Group 3 - joint pains & 185 & 14.5 \\
Group 4 - gastrointestinal disturbances & 211 & 14.5 \\
Total & 746 & 22.5 \\
\hline
\end{tabular}

- Group 4: gastrointestinal disturbances - including problems of the liver, gall bladder, and pancreas - in patients over 20 years of age. This survey was carried out in November, 1993.

In the cross sectional surveys, only one contact record form was filled in per patient. Visits were grouped according to the specific complaint and whether this was a first consultation or a repeat (follow up). Patients who contacted the doctor more than once within the reporting period were identified by means of their date of birth, sex, diagnosis, and the reason for the visit. Only the first contact remained in the data: further contacts were eliminated.

Some of the EVaS study methodology ${ }^{7}$ was adapted to design complementary standardised questionnaires to ascertain the following variables:

- A general practice questionnaire, which included items on physician and office variables; conditions under which physicians conduct their practice; personnel and technological equipment; the physicians' attitudes towards the 1993 reform act.

- A contact report form, which detailed patient management in the individual practices; documentation of a sample of consultations related to various health problems (as expressed by the patient). This survey instrument records sociodemographic variables and the patient's major reasons for the visit. Furthermore, it gives insight into diagnostic and treatment strategies according to the physician's diagnosis and his or her estimation of the severity of the patient's problem. Several items on the patient record form stress the physician's treatment changes and reasons for these changes (for example, medical reason, cost containment, inclusion of a drug on the "negative" list). The study focussed on the practitioners' individual treatment of patients with different reasons for the visit. There was no collection of morbidity data (that is prevalences) in contrast with epidemiological surveillance programmes. For this reason validation of the physicians' diagnoses is not necessary within this study.

Altogether physicians responded to the general practice questionnaire throughout the year. The validity of the data derived from this questionnaire was checked by means of important variables (age, sex, date of setting up practice) from the data the practitioners' association made available. During the year, a total of 3728 contact report forms were completed in full by the participants. Table 1 summarises the data collected according to the four different surveys. Table 2 shows the sex distribution of the patients included in the study.

\section{Results}

The influence of the health care reforms below are related to follow up patients - that is, those who consult the doctor on more than one occasion about the same health problem. In 
$31 \%$ of all contacts there was a change in the treatment strategy. Doctors explained $24 \%$ of these changes in terms of cost containment. The rates differed considerably, however, between four surveys (see table 3 ). These differences might be explained, firstly, by the physicians' adaptation to the cost containment policies within the year and, secondly, in terms of the disease complex involved.

We investigated the highest number of treatment changes induced by the reform act in the first survey - that involving the group 1 complex vertigo, low blood pressure, fatigue, insomnia, disorders of coordination, restlessness, anxiety, and loss of memory. This complex may have a major psychosomatic component in comparison with the other three symptom complexes, which have more physical relevance (leg problems, chronic joint pains, and gastrointestinal disturbances). We could not explain all the differences in terms of the physicians' adaptation to the health reform act within the year, because the three more physical disease complexes varied only slightly with regard to the frequency of changes in treatment as a result of cost containment.

The data showed that the percentage of treatment changes resulting from cost containment policies was highest for the diagnosis "hypotension" within the complex group 1 vertigo etc: $59 \%$ of all changes in treatment of hypotension were attributed to the 1993 reform law.

Furthermore, the data reflect the fact that physicians who had previously been penalised financially (Regreßpflicht) before 1993 because they had not conformed to "economic prescribing" more often changed their treatment strategy to achieve cost containment (odds ratio 3.45 as estimated by various multivariate logistic regression models).

In addition to the frequency of changes in the physicians' treatment strategy, their attitudes towards the qualitative effects of the health reforms are of interest. The documentation of changes in treatment included in the physicians' prognosis as to whether the:

- Therapeutic benefit;

- Adverse effects;

- Patient satisfaction

would increase, decrease, or remain unchanged after starting the new treatment. According to these three determinants we were able to make a hypothetical comparison of the new and the prior treatment from the physicians' point of view.

The data showed that when cost containment played a role in the treatment change, the physicians' prediction of the outcome was mostly neutral or positive: only a few cases were judged likely to be negative. For instance, physicians felt that the therapeutic benefit would decrease in $17 \%$ of group 2 - "leg problems" with such a change in treatment (group 1 - "vertigo" $4 \%$, group 3 - "joint pains" $8 \%$, group 4 - "gastrointestinal disturbances" $3 \%)$. Other reasons for the change were regarded as neutral or likely to lead to an improvement in the treatment benefit.

Analysis of the physicians' prognoses of ad- verse drug effects showed similar results with regard to reform-induced treatment changes. When other reasons played a role in changing the treatment strategy, however, physicians expected the development of adverse drug effects to decrease in some cases, too.

From the physicians' point of view patient satisfaction was the criterion which suffered most as a result of the 1993 reforms. When the treatment regimen was changed as a result of cost containment, a reduction in patient satisfaction was predicted by the physicians in $26 \%$ of all changes associated with group $2-$ "leg problems" (group 3 - "joint pains" 15\%, group 4 - "gastrointestinal disturbances $14 \%$ ). Again, other reasons for a therapeutic change were usually forecast to increase the patients' satisfaction.

According to the doctors, patients who considered their own health problem to be overly serious more often had their treatment changed as a result of the 1993 reform law than patients who saw their problem as less serious. Thus, $6 \%$ of the patients in a "less serious" complaint had a change in treatment compared with $15 \%$ of the patients with a "more serious" complaint.

One explanation for this may be greater consideration for patients' emotional problems on the part of the physicians before the countrywide implementation of the drug budget. After the 1993 reform law, physicians were financially penalised if they did not prescribe cost effectively, thus giving them an incentive to provide clinically oriented, less emotionally supportive care. The physicians' prognosis about the development of the three determinants of care quality did not differ, however, between these two groups of patients.

\section{Discussion}

The German health care system, unlike the British or Dutch system, is based on unrestricted access to any practitioner, including specialists. One of the major problems of sentinel research in Germany is the absence of patient lists or registers describing the population covered by each practice. External validation of patient data derived from sentinel practice networks is not possible because of the German ambulatory care system. In this study, validation of the practice data was ensured by data from the associations of sickness fund physicians. Validation of the diagnoses was not necessary for the purposes of the study.

Bias might be introduced in one of the following ways:

- The participating practitioners might have different characteristics from non-participating physicians. The very fact of participating in this sentinel study is indicative of a special motivation. It cannot be assumed that the responders represent the entire profession in the study region, but we did find that the distribution of several variables (age, sex, practice speciality, location of practices (urban or rural)) for the study population was quite close to that of all primary care practitioners in Lower Saxony. 
- Selection bias may also be introduced by the data collection. Physicians might have selected patients for participation in the study within the reporting period. Because of the absence of patient lists or routinely available electronic data this problem of undercoverage cannot be controlled for - it is a product of Germany's health care system.

- Selection bias might also result from some physicians reporting, in particular, those consultations in which therapy changes were made. A sensitivity analysis which eliminating data from five physicians who assigned the reason for more than $50 \%$ of their therapeutic changes to the 1993 reforms showed that although the frequency of treatment changes (with respect to the health reforms) was reduced, the results regarding physicians' qualitative assessments towards the new treatment remained stable.

Patient satisfaction is one important determinant of the quality of Germany's ambulatory health care system, which is based on competition and characterised by free access to any physician. In this study, patient satisfaction was intentionally estimated by the physician. This improved the practicability of the study and acceptance by the participating physicians and was not an unrealistic demand since in Germany, ambulatory care providers are required to be capable of assessing the satisfaction of their patients. Physicians' practices, unlike in the UK or the Netherlands, depend on the satisfaction of their "consumers". For this reason we consider the bias derived from this kind of measurement to be low.
When doctors changed the treatment regimen because of the 1993 reforms it was predicted that the patients' satisfaction with ambulatory care would suffer in some cases. Only a minority of the changes related to cost containment were judged by the doctors to reduce the treatment benefit. There was an improvement in patient outcome recorded in some cases, too. A possible explanation might be that unnecessary treatments (for example, for hypotension) have been cut out because of the reforms. On the whole, the 1993 reforms are likely to be neutral towards patient outcome.

Furthermore, the data showed that there are groups of patients and groups of physicians who are specifically concerned regarding the 1993 reforms. For example, physicians who had experienced monetary restitution before 1993 were influenced more strongly by the reform law than their peers.

In conclusion, it is noticeable that the physician's changed their assessments with regard to the reform act in the course of 1993. Sentinel practice networks were found to be suitable to work on cost containment policies.

1 Lambird PA. Resource allocation and the cost of quality. Arch Pathol Lab Med 1990;144:1168-72.

2 Reiners H. Das Gesundheits-Reformgesetz - Ende der Kostenexplosion im Gesundheitswesen? Arbeits-u Sozialpolitik 1990;1:39-42.

3 Seehofer H. Die nächste Gesundheitsreform. Politische Mornung 1993;38(2):51-5.

4 Knox R. Germany's healty system. Washington; Faulkner and Gray, 1993:183.

5 Royal College of General Practitioner. Collective research in general practice influenza. $\mathcal{F} R$ Coll Gen Pract 1977;27 544-51.

6 Collete RJA. The sentinel practices system in the Netherlands In: Leaverton PE ed. Environment epidemiology. New York: Praeger, 1982:149-55.

7 Schwartz FW, Schach E, Kerek-Bodden HE. Die EVaS Studie. Köln: Deutscher Ärzte Verlag, 1989. 